\title{
Wavelet-FFT Filter Applied to Non Uniformity Correction in Infrared Imaging System
}

\author{
Cesar San Martin ${ }^{1,2}$, Carlos Deocares ${ }^{1,2}, \mathrm{~S}$. Godoy $^{2}$, P. Meza $^{2}$, and Daniela Bonilla ${ }^{1,2}$ \\ ${ }^{1}$ Information Processing Laboratory, Electrical Engineering Department, \\ Universidad de La Frontera, Casilla 54-D, Temuco Chile \\ cesarsanmartin@ufro.cl \\ ${ }^{2}$ Center for Optics and Photonics, Chile
}

\begin{abstract}
In this paper, we use the recently presented wavelet-FFT filter [1] to reduce the nonuniformity noise that affect almost all infrared imaging systems. The wavelet-FFT filter was originally developed to compensate the onedimensional noise known as stripping noise. We perform an extension of this methodology in order to compensate the two-dimensional noise that degrades infrared imagery. The principal hypothesis of this work is that the twodimensional focal-plane array can be considered as the composition of vertical and horizontal one-dimensional array sensors. Under this assumption we use a specific design of the wavelet filter to synthesize a replica of the twodimensional noise and then recover the real incident radiation. The method is evaluated using real mid- and long-wave infrared data from two cameras. The results show the promising performance of the wavelet-FFT filter when is applied in infrared imaging system such as self heating effect.
\end{abstract}

\section{Introduction}

The noise that corrupts the one- and two-dimensional imaging systems is an artifact common to all the focal-plane array-based imaging systems. This noise becomes more important in systems that acquire infrared (IR) radiation due to the low level of radiant energy collected by the sensors. In addition, the IR detectors in a focal-plane array (FPA) normally have physical differences even though they are fabricated under the same specifications. These differences give rise to a no uniform photo response in the sensors array that creates a fixed-pattern noise (FPN). The principal characteristic of this noise is that the pattern does not change significantly in time [2]. The sensors array can be found in both one- or two-dimensional form. If we have a horizontal (vertical) one-dimensional array of sensors, the images are created performing a vertical (horizontal) scanning. Considering this fact, the FPN present in the raw data is called stripping noise because it can be seen as vertical (horizontal) lines across the image, [3]. The one-dimensional arrays are typically used in remote sensing technology, satellite applications, spectroradiometry, multi- and hyper-spectral images, etc. For two-dimensional arrays, the FPN is normally referred in the literature as the nonuniformity (NU) of noise. As was aforementioned, the that NU noise is presented in 
IR imaging technology mainly due to the low energy presented at 3-5 um (mid-wave) and 8-14 um (long-wave) wavelengths IR ranges. The reduction of the stripping noise is an active area in the digital image processing field [3-5] due to their applications in several scientific studies. On the other hand, nonuniformity correction (NUC) is an active research field that is normally classified in two subareas of development: reference- and scene-based methods. The reference-based methods require a flat field of radiation emitted from black-bodies at different temperatures. Using this uniformand-known input as reference, we can obtain a direct relationship between the reference and the readout data. The relationship allows us to estimate the two-dimensional noise in a very accurate way, which is desirable for thermographics applications. Depending of the number of measurement considered the method is called one-, two- or multi-point calibrations. Nonetheless these methods are accurate, simple and reliable; the references are not always available due to the high cost of black body radiators [6]. In the scene-based method, the NUC can be performed using mathematical or statistical operations in time, no requiring halt the normal operation of the camera. Several examples can be seen in $[2,7,8]$. Some methods work in the pixel basis, and others assume some spatial relations between pixel neighborhoods.

Munch et al. in [1] report a fast and stable filter based on wavelet and Fourier transform, that they called wavelet-FFT filter. Briefly, this method has two key steps: wavelet decomposition and damping process. The first one permits to separate the information into horizontal, vertical and diagonal details in several scales. For vertical stripping noise, the vertical detail band contains the principal stripe artifacts. Therefore the Fourier transform is applied only in this band in order to reduce the stripping noise by means of a Gaussian high-pass filter. The de-stripe image is then reconstructed using the inverse wavelet transform. In order to tune the performance of the filter, we can adjust two parameters: decomposition level of wavelet transform, and the damping factor of the high pass filter. The decomposition level allows us to select the frequency band where the filter is applied and the damping factor modifies the filter bandwidth. The lower the damping factor, the higher the bandwidth.

In this paper, we use the wavelet-FFT filter to perform NUC for IR imaging system. The principal assumption of this work is that the two-dimensional FPA can be considered as the composition of vertical and horizontal one-dimensional arrays. Under this assumption we select the best decomposition alternative, generating two stripping-noises-like images from the decomposed virtual arrays. We applied the wavelet-FFT to this virtual 1D array to denoise the readout data. With the noise-free imagery, we estimate the real two-dimensional noise in order to denoise the following frames in the image sequence. The performance of the metric is evaluated using real data in mid- and long-IR wavelength range and compares with state-of-art NUC methods.

\section{Wavelet-FFT Filter as NUC Method}

The typical mathematical model used to describe the input-output relationship for each sensor in a FPA is given by: 


$$
Y(n, m)=A(n, m) I(n, m)+B(n, m),
$$

where $A(n, m)$ and $B(n, m)$ are the gain and offset of the $(n, m)-t h$ pixel in the array. $I(n, m)$ is the input irradiance and $Y(n, m)$ is the readout signal for the same pixel. Gain and offset summarize the principal factors that generate the no uniform photo response in IR FPA sensors [6]. The result of these no uniform parameters gives rise to the NU noise present in IR images. This mathematical model represents both the $1 \mathrm{D}$ and the 2D sensors array, with the difference that for 1D arrays, one of the spatial coordinates must be set equal to unity: $n=1$ for horizontal arrays or $m=1$ for vertical arrays. To generate the 2D image from a 1D array the scene must be scanned in time by moving the target or the camera. This implies that a horizontal array will generate a vertical stripping noise, and the gain and offset from eq. (1) will be given by:

$$
\begin{aligned}
& A(n, m)=A_{m} \delta(m), \\
& B(n, m)=B_{m} \delta(m),
\end{aligned}
$$

presenting variations only in the coordinate $m$. The principal characteristic of the stripping noise is that, in the Fourier domain, it concentrates the vertical (horizontal) stripes in horizontal (vertical) frequency components. In fact, taking the Fourier transform to eq. (2) and (3) we obtain:

$$
\begin{gathered}
F A(u, v)=\sum_{m=0}^{M-1} A_{n} e^{-j m v} \delta(u), \\
F B(u, v)=\sum_{m=0}^{M-1} B_{m} e^{-j m v} \delta(u) .
\end{gathered}
$$

Using this results in the frequency-domain of the stripping noise, a wavelet-FFT filter was proposed in [1] in order to improve the quality of the raw image $Y(n, m)$. Let us refer to eq. (1). In the frequency domain, the offset is located only for $u=0$, which is the easiest case and was evaluated in the corresponding reference, [1]. When we face a problem that includes gain -as in our case-- more elaborated analysis must be done. Recall that in the frequency-domain the image corrupted by the nonuniform gain is given by the convolution between the Fourier transform of the gain and the Fourier transform of the image. This implies that the gain noise is not concentrated in any frequency, but is dispersed around $u=0$. Indeed, the noise produced by the gain has a bandwidth that is commonly called stripping frequency band (SFB) that can also include the offset effects. Based on this analysis, it is possible to postulate that a simple way to cancel the stripping noise is by the reduction of the SFB. In order to perform this we can use a filter with the form [1]:

$$
g(u)=1-e^{-\frac{u^{2}}{D^{2}}},
$$

where $D$ is the damping factor that is selected accordingly with the SFB. 
The well-known wavelet transform allow us to separate an image in four images (first decomposition level): diagonal, vertical and horizontal details and the approximation image. If the multi-resolution algorithm is applied, consecutively the approximation images are decomposed in another four images. Then, the filter $g$ is applied only in vertical details, which gives an improvement of the filter performance. As a consequence, the wavelet-FFT filter requires the adjustment of two parameters: the damping factor and the decomposition level of the wavelet transform. The best pair of values that improve the filter performance are obtained by trial-and-error. The effectiveness of the filter is given by the resolution in the frequency domain: the more the information in the vertical direction, the more effective is the use of the filter given by eq. (6).

\subsection{Realignment of 2D FPA into 1D FPA}

As was aforementioned, the principal assumption of this work is that the twodimensional FPA can be decomposed in two one-dimensional virtual arrays. The procedure to realignment the 2D FPA is addressed in this section. Let us consider a $\mathrm{N} \times \mathrm{M}$ FPA sensor. Since the frames are captures at discrete-time basis we can capture a block of $\mathrm{K}$ frames, forming a tree-dimensional matrix of data, as it is shown in the Fig. 1.

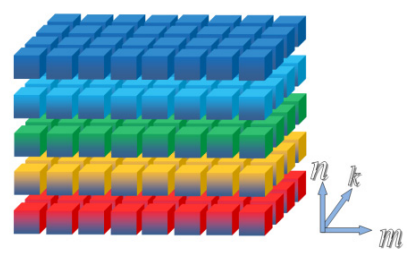

Fig. 1. Constructions of $\mathrm{N} \times \mathrm{M} \times \mathrm{K}$ variable in which the rows are the $n$-positions, the columns are denoted by $m$ and $k$ is sample time o frame number. Each row has different color (from up to down): blue, cyan, green, orange and red; representing each image with stripe noise.

During the last years, the nonuniformity has been well documented and its principal characteristic is that presents fixed-patterns during blocks of time [2, 7, 8]. Under this scenario, we can observe that the patters present among rows (columns) are fixed during a block of $\mathrm{K}$ frames, so stripping-noise-like images can be obtained from a row-to-row (column-to-column) analysis across time. Let us be focused on the rowto-row analysis. For each row in the Fig. 1, we consider only $m$ and $k$ dimensions, in order to generate $\mathrm{N}$ images of $\mathrm{M} \times \mathrm{K}$ virtual pixels. Therefore, each image given by a different color in Fig. 1, can be considered as images captured by one-dimensional virtual FPAs with M detectors. The wavelet-FFT filter previously described is applied to each one of these virtually generated images, and the one-dimensional-compensated block is restored again in the real $\mathrm{N} \times \mathrm{M} \times \mathrm{K}$ block of data. Further, for each column the process is repeated but considering that the striping-noise--like images are obtained in $n$ and $k$ directions generating $\mathrm{M}$ images captured by a onedimensional virtual FPA with $\mathrm{N}$ detectors. 


\section{Results}

In this section, the proposed method is evaluated and compared with two state-of-art NUC methodologies using real infrared data from two different cameras that are corrupted by two-dimensional noise. This comparison is made based on both classical metrics that we present below and naked-eye evaluation of the final results. For this paper, in all cases Daubechies basis has been fixed as wavelet function with 12 coefficients in order to obtain a first approach of the methodology.

\subsection{Metrics}

Two quality metrics are used in this paper with comparison purposes. The first one corresponds to the root-mean-square error (RMSE) given by:

$$
R M S E^{2}=\frac{1}{N M} \sum_{i} \sum_{j}(I(i, j)-X(i, j))^{2},
$$

where $I$ is the real image (reference image) and $X$ is the corrected image with the NUC method under study. The RMSE require the knowledge of the real incident infrared radiation, which in our case can be approximated with a black-bodycorrected image [2].

Nonetheless, in some cases this reference is not disposable, becoming necessary a reference-free quality index. A good example of this is the roughness index given by:

$$
\rho=\frac{\|h \otimes X\|_{2}+\left\|h^{T} \otimes X\right\|_{2}}{\|X\|_{2}}
$$

where $h$ is a 1D high pass filter, $T$ transpose and $\otimes$ denote convolution. The comparison of the roughness index must be used in conjunction with naked-eye evaluation because it only shows if the correction is producing a smooth image or not, leaving out any radiometric modifications.

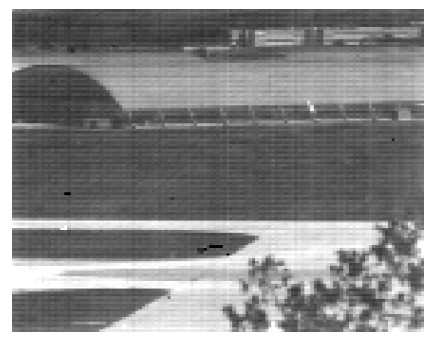

a)

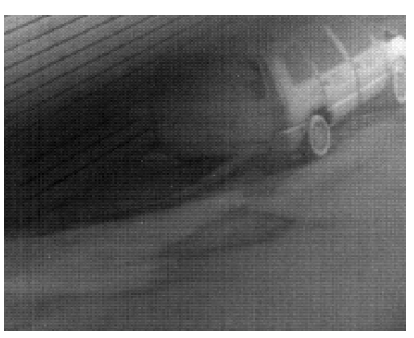

b)

Fig. 2. Example of IR data used in this work: a) mid-wave IR image, and b) long-wave IR image 


\subsection{Infrared Data}

In this paper two real infrared data has been used to evaluate the proposed method. The first sequence has been collected using a 128×128 InSb FPA cooled camera (Amber Model AE-4128) operating in the 3-5 um range. An example of the readout data of this camera is depicted in Fig. 2(a). For this dataset, data of black-body radiators is available, so the quantity $I(i, j)$ can be obtained using a two-point calibration method. The second sequence of infrared data has been recorded using a $320 \times 240$ $\mathrm{HgCdTe}$ FPA cooled camera (CEDIP Jade) operating in the $8-12$ um range. An example of the readout data of this camera is depicted in Fig. 2(b). Since we do not have black-body data or this dataset, only roughness index is used as comparison.

\subsection{Results}

In the Fig. 3 we resume the steps used in this section, accordingly with the afore explained procedure. The first step of the proposed method is build the $\mathrm{N} \times \mathrm{M} \times \mathrm{K}$ variable (Fig. 1) using a sequence of consecutive frames as shown in Fig. 3a. The aim is to obtain image with stripping noise (Fig. 3b). The wavelet-FFT filter is applied over each one of these images, obtaining a corrected version (Fig. 3c). Further, transposing the 2D FPA, new image with vertical stripping noise is obtained, and the process is repeated.

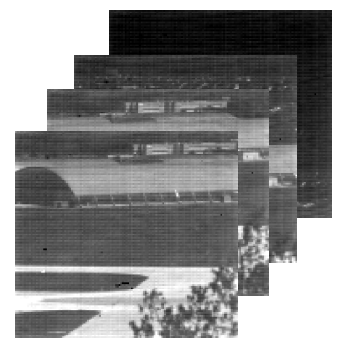

(a)

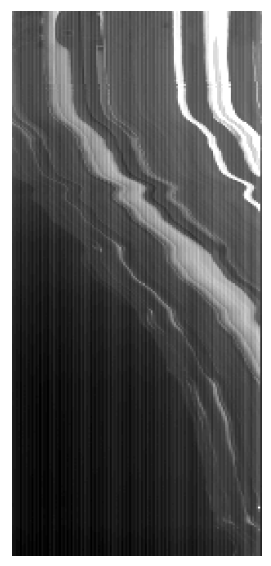

(b)

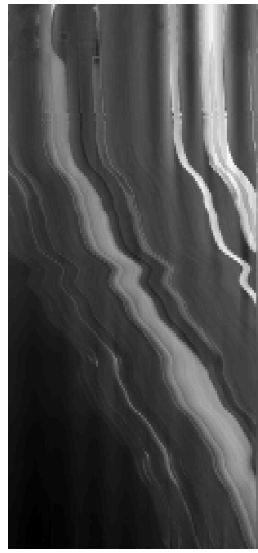

(c)

Fig. 3. Realignment of the 2D FPA to 1D FPA. (a) shows the construction of the variable presented in Fig. 1 ; b) shows the image with stripe noise; and c) is the corrected image using wavelet-FFT filter.

Some results of this methodology can be observed in Fig. 4. The corrupted image (readout data) is presented in Fig. 4a. Fig. 4b is the corrected version of the same image using the proposed method. Fig. $4 \mathrm{c}$ and $4 \mathrm{~d}$ are the corresponding corrected frame using the statistical algorithm proposed in [8] and the Kalman filter proposed in [2]. This method use a block of frame in order to obtain the gain and offset, and then, 
the NUC is performed. Note that the proposed method is based in the use of a block of frames in order to build the image with stripping noise. In the Fig. 4 is easy to see that the proposed method presents a comparative behavior with other techniques. In order to compare the performance of those methods as function of the length of the block of frames, six values are selected. For all of these cases the RMSE is computed using the corrected data from black-body radiators. Table 1 resumes the principal results. Clearly, the proposed method exhibits the best values for the RMSE. This is due to the fact that the wavelet-FFT filter maintains the radiometric range of the original imagery.

Note that the RMSE is actually an average RMSE over all pixels in a frame. Of course, the lower the value of RMSE, the better the NUC achieved.

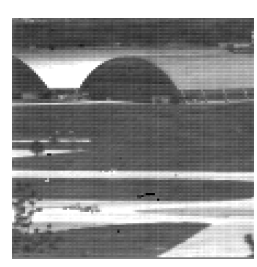

a)

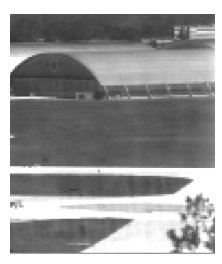

b)

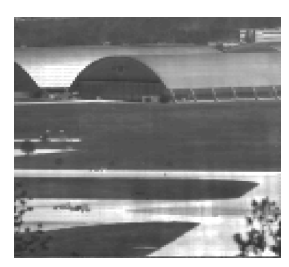

c)

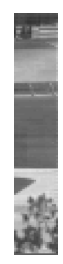

d)

Fig. 4. Results using the mid-wave IR data: a) corrupted data, b) corrected using the proposed method; c) statistical algorithm and d) Kalman filtering. Comparative correction is obtained using the wavelet-FFT in same sequence.

The second experiment considers data from long-wave infrared camera (Fig. 2b). Table 2 resumes the results using the roughness parameter. Clearly the performance of the proposed method is consistent with the performance of statistical algorithm and Kalman filter. But, using the naked-eye analysis in a particular frame (Fig. 5) the proposed method generates lower ghosting artifacts than the other methods under study. Based on a subjective analysis over the image contrast, from Fig. $5 \mathrm{~b}$ is possible to conclude that the method preserve the radiometric range. Fig. 5c and 5d do not show the same behavior. Additionally, the ghosting generated by the car presented in Fig. $2 b$ is showed in both images, whereas it is reduced in Fig. 5b.

Table 1. RMSE using three NUC methods: statistical algorithm, Kalman filter and the proposed method. The RMSE is calculated for 1000 frames.

\begin{tabular}{cccc}
\hline Frames & Statistical algorithm & Kalman filter & Proposed method \\
\hline 50 & 5.4963 & 3.1459 & 0.6494 \\
100 & 5.3372 & 2.8570 & 0.6097 \\
150 & 5.3320 & 2.6561 & 0.6427 \\
200 & 5.3372 & 2.7410 & 0.6392 \\
250 & 5.4779 & 2.7600 & 0.6627 \\
300 & 5.3322 & 2.8668 & 0.6378 \\
\hline
\end{tabular}




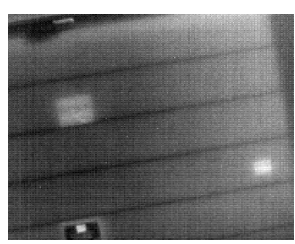

a)

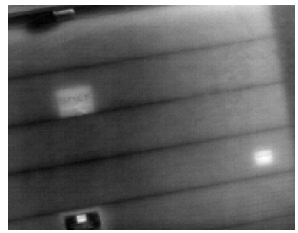

b)

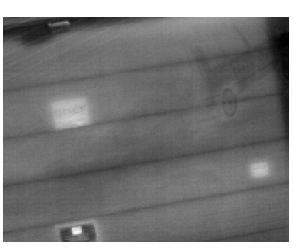

c)

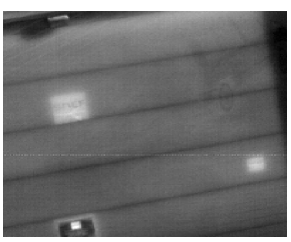

d)

Fig. 5. Results using the long-wave IR data: a) corrupted data, b) corrected using the proposed method; c) statistical algorithm and d) Kalman filtering. Note that in the proposed method no ghosting effect is presented.

Table 2. Roughness index using three NUC methods: statistical algorithm, Kalman filter and the proposed method. The roughness index is calculated for 1000 frames.

\begin{tabular}{rccc}
\hline Frames & Statistical algorithm & Kalman filter & Proposed method \\
\hline 25 & 0.6622 & 0.6866 & 0.9892 \\
50 & 0.7038 & 0.7022 & 0.7296 \\
100 & 0.7038 & 0.7185 & 0.7184 \\
150 & 0.7125 & 0.7213 & 0.7087 \\
\hline
\end{tabular}

\section{Conclusions and Future Work}

In this paper, a wavelet-FFT filter is adapted to reduce FPN in infrared imaging system. The principal hypothesis is that the 2D FPA can be decomposed in two 1D FPA, and then, a de-stripping algorithm is applied. The performance of the method has been evaluated using two real IR sequences: mid and long wave IR data. In both cases, the performance of the method is compared with two techniques presenting a similar behavior. The proposed method exhibit two advantages: i) the radiometric range is preserved and ii) the method generate reduced ghosting artifact. Then, the waveletFFT filter is a promising tool to apply in NUC problems. The future work include more numerical experiments and analysis using real IR data specially when ghosting is presented in thermal images considering the influence of the kind of wavelet used.

Acknowledgements. This work was partially supported by Proyecto DI-UFRO DI120064 and Center for Optics and Photonics CEFOP FB0824/2008. The authors wish to thank Ernest E. Armstrong (OptiMetrics Inc., USA) and Pierre Potet (CEDIP Infrared Systems) for collecting the data, and the United States Air Force Research Laboratory, Ohio, USA.

\section{References}

1. Munch, B., Trtik, P., Marone, F., Stampanoni, M.: Stripe and ring artifact removal with combined wavelet-Fourier filtering. Opt. Express 17, 8567-8591 (2009)

2. Torres, S., Hayat, M.: Kalman Filtering for Adaptive Non uniformity Correction in Infrared Focal Plane Arrays. The JOSA-A Opt. Soc. of America 20, 470-480 (2003) 
3. Liu, J.G., Morgan, G.L.K.: FFT Selective and Adaptive Filtering for Removal of Systematic Noise in ETM+Imageodesy Images. IEEE Trans. Geosci. Remote Sens. 44, 3716-3724 (2006)

4. Zhang, Z., Shi, Z., Guo, W., Huang, S.: Adaptively Image De-striping through Frequency Filtering. In: ICO20: Opt. Inf. Proc., Proc. SPIE 6027, p. 60273V (2006)

5. Wang, Z., Fu, Y.: Frequency-domain Regularized Deconvolution for Images with Stripe Noise. In: ICIG Proc. 4th Intl Conf. Image and Graphics, pp. 110-115 (2007)

6. Godoy, S., Torres, S., Pezoa, J., Hayat, M., Wang, Q.: Nonuniformity correction algorithm based on a noise-cancellation system for infrared focal-plane arrays. In: Proc. SPIE, vol. 6542 (2007) $65423 \mathrm{~S}$

7. Torres, S., Pezoa, J., Hayat, M.: Scene-based Nonuniformity Correction for Focal Plane Arrays Using the Method of the Inverse Covariance Form. OSA App. Opt. Inf. Proc. 42, 5872-5881 (2003)

8. Hayat, M., Torres, S., Amstrong, E., Cain, S., Yasuda, B.: Statistical algorithm for non uniformity correction in focal plane arrays. Applied Optics 38, 773-780 (1999) 